Fields Institute Communications

Volume 00, 0000

\title{
Competition for Essential Resources: A brief review
}

\author{
Hal L. Smith \\ Department of Mathematics \\ Arizona State University \\ Tempe, AZ 85287-1804 \\ Bingtuan Li \\ Department of Mathematics \\ University of Louisville \\ Louisville, KY 40292
}

This paper is dedicated to Paul Waltman on the occasion of his 70th birthday

\begin{abstract}
We review, and in some cases extend, results on the standard model of $n$ species competing for $m$ essential resources using the law of the minimum functional response.
\end{abstract}

\section{Introduction.}

Renewed interest in the area of resource competition has been recently triggered by results of numerical simulations by Huisman and Weissing [19, 20] of the standard model of $n$ species competing for $m$ essential resources, using the Law of the Minimum. Even a popular science publication has taken note (see [3])). Simulations in [19] suggest that, for certain parameter values, coexistence may occur in the form of a periodic orbit for $n=m=3$ and as a strange attractor for $n=m=5$ and as many as nine species can seemingly coexist on three resources and as many as twelve species can coexist on five resources. Simulations in [20] suggest that for certain parameters in the case $n=5, m=3$ there exists two limit cycles, one involving only the first three species, the other involves only species one,four and five, and that the basins of attraction of the two limit cycles are highly interwoven, with possible fractal boundaries. This leads to extreme sensitivity of the outcome of competition, which species survive, on initial data. These results have been somewhat controversial for a number of reasons. First, their essential novelty has been questioned $[28,21]$. Second, due to the well known tendency in competition models for an eventual loser to hang around for very long time periods, one cannot be sure one has integrated long enough. Finally, it is not clear whether the range

1991 Mathematics Subject Classification. Primary 92A15, 34C25.

The first author was supported in part by NSF Grant \#0107160. 
of parameters for which these sort of outcomes may occur represent a significant region of biologically realistic ones.

Smale [31] showed in principle that essentially any $(n-1)$-dimensional dynamics was possible for Kolmogorov-type competition models of $n$ species. Armstrong and McGehee certainly pointed out the possibility of complicated behavior as well as the failure of the competitive exclusion principle in its most naive form, namely that $n$ species cannot coexist on fewer than $n$ resources, in their widely cited paper [1], still interesting to read even now for its early formulation of the idea of species persistence.

However, the cautionary warning given by these authors to population biologists on the potential for complicated dynamics in systems of differential equations modeling competition was largely based on the behavior of mathematically contrived systems which satisfied only the crudest mathematical prerequisites for being a competition model. By contrast, Huisman and Weissing's simulations are carried out for the "standard model" of resource competition, long favored by biologists studying algae populations in aquatic environments $[9,35]$. Clearly, the warning must be taken seriously.

Mathematically rigorous results for the standard model exist for small values of $m$ and/or $n$. Results for the case $m=1$, the classical chemostat equations of competition for a single resource, are extensive and nearly complete [37, 24, 25, 18, 16]. See [32] for a review. The case $m=n=2$ has been completely settled, first by Leon and Tumpson [23] and later with more mathematical precision by Hsu, Cheng and Hubbell [17] and Butler and Wolkowicz [5]. Our recent work in [26] settled the case $m=2, n=3$ (but not $m=2$ and larger $n$ ) and the work of the second author in [27] established the existence of the limit cycle for $n=m=3$ which is fundamental to many of the conclusions of Huisman and Weissing. Our work exploits the Poincaré-Bendixson theory for three dimensional competitive systems and work of Hirsch $[11,12]$.

It is our intention in this paper to provide a review of results for the standard model with emphasis on our own most recent work. Many of our results will carry over to more general functional response. Before specializing to particular $m$ and $n$, we show that a great deal can be said in the general case. [22] appeared as final revisions to this paper were being made following its acceptance; it offers a review from a more biological perspective.

\section{The Standard Model and general considerations}

The equations for $n$ species $N_{i}$ competing for $m$ resources $R_{j}$ are

$$
\begin{aligned}
& N_{i}^{\prime}=N_{i}\left[\mu_{i}(R)-m_{i}\right], 1 \leq i \leq n \\
& R_{j}^{\prime}=D\left[S_{j}-R_{j}\right]-\sum_{k} c_{j k} \mu_{k}(R) N_{k}, 1 \leq j \leq m .
\end{aligned}
$$

Here, the removal rate of the $i$ th species is $m_{i}>0$, resource turnover rate is $D>0$ and supply of the $j$ th resource is $S_{j}>0$. On re-scaling the $N_{i}$, we can assume that that the $c_{j i}>0$ satisfy $\sum_{j} c_{j i}=1 ; c_{j k}$ is the fraction of species $k$ consisting of resource $j$.

We restrict attention to essential resources $R$ for which the Law of the Minimum applies

$$
\mu_{i}(R)=\min _{j}\left\{f_{j i}\left(R_{j}\right)\right\}
$$


where, typically, $f_{j i}$ is a Monod function

$$
f_{j i}\left(R_{j}\right)=\frac{r_{i} R_{j}}{K_{j i}+R_{j}},
$$

although we really require only that $f_{j i}$ is continuously differentiable and that

$$
f_{j i}(0)=0, f_{j i}^{\prime}\left(R_{j}\right)>0 \text { for } R_{j} \geq 0 .
$$

See [8] for a derivation of (2.2) as well as other growth functions. We emphasize the assumption that each resource is an essential requirement for growth of every one of the species.

Very little progress has been made in the case that the $m_{i}$ are distinct (although see S.B. Hsu [16], B. Li [24, 25] and Wolkowicz and $\mathrm{Lu}[37]$ in case $m=1$ ). We assume hereafter that

$$
m_{1}=m_{2}=\cdots=m_{n} \equiv D
$$

because this leads to a substantial reduction in dimension. In this case, (2.1) takes the form

$$
\begin{aligned}
N^{\prime} & =G-D N \\
R^{\prime} & =D(S-R)-C G
\end{aligned}
$$

where $G=\left(N_{1} \mu_{i}(R), \cdots, N_{n} \mu_{n}(R)\right)^{T}$. Multiplying the first equation by $C=\left(c_{j i}\right)$ and adding to the second equation, we have

$$
(R+C N)^{\prime}=D[S-(R+C N)]
$$

which leads to

$$
(R+C N)(t)=(R+C N)(0) e^{-D t}+S\left(1-e^{-D t}\right) .
$$

Write $c^{i}$ for the $i$-th column of $C$, and note that column sums of $C$ are one. We will consistently put the resource subscript first and the species subscript second. The above integration establishes the existence of an invariant, exponentially attracting, affine sub-manifold.

$$
M \equiv\left\{(R, N) \in R_{+}^{m+n}: R+C N=S\right\}
$$

Remark 2.1 Because all column sums of $C$ are one we find that $1_{m}^{T} C=1_{n}^{T}$. Here, $1_{p}$ is the $p$-vector of ones. As all omega limit points $(\tilde{R}, \tilde{N})$ of a solution of (2.5) satisfy $C \tilde{N}+\tilde{R}=S$, we find on multiplying by $1_{m}^{T}$, that

$$
\sum_{i=1}^{n} \tilde{N}_{i}+\sum_{j=1}^{m} \tilde{R}_{j}=\sum_{j=1}^{m} S_{j} .
$$

Equivalently, resource plus biomass (in resource equivalent units) must equal resource in the feed, asymptotically as $t \rightarrow \infty$.

The dynamics restricted to $M$ are given by

$$
N_{i}^{\prime}=N_{i}\left[\mu_{i}(R)-D\right], R=S-C N, 1 \leq i \leq n .
$$

on the positively invariant polygonal set

$$
\Gamma \equiv\left\{N \in R_{+}^{n}: C N \leq S\right\} .
$$

Inequalities between vectors are to be interpreted component-wise. We write $v \leq w$ if $v_{i} \leq w_{i}$ for all $i$ and $v<w$ if $v_{i}<w_{i}$ for all $i$.

It will be useful later on to extend (2.8) to all of $R_{+}^{n}$ in such a way that the time-reversed flow is strongly monotone (see Lemma 2.6 below). For this reason, 
we assume, without loss of generality, that each $f_{j i}$ extends as a continuously differentiable, strictly increasing function on $(-\infty, \infty)$ with bounded derivative on $(-\infty, 0]$. In particular, $f_{j i}\left(R_{j}\right)<0$ for $R_{j}<0$. This has the harmless effect that a solution of (2.8) initiating at point $N(0) \in R_{+}^{n} \backslash \Gamma$ satisfies $N(t) \leq N(0) e^{-D t}$ until it reaches $\Gamma$. Later on we return to examine this limiting system (2.8). For now, we focus attention on the original system (2.5).

We assume that there exists positive real numbers $\lambda_{j i}$ such that

$$
f_{j i}\left(R_{j}\right)-D<0,0 \leq R_{j}<\lambda_{j i}, \quad f_{j i}\left(R_{j}\right)-D>0, R_{j}>\lambda_{j i} .
$$

Thus, $\lambda_{j i}$ is the break-even concentration of resource $R_{j}$ for the growth of species $i$ when only resource $R_{j}$ is growth limiting. Denote by $\lambda=\left(\lambda_{j i}\right)$ and $\lambda^{i}$ the $i$ th column of $\lambda$.

Let $Q_{i}$ denote the "isocline" (in resource space) for the $i$-th species:

$$
Q_{i}=\left\{R \in R_{+}^{m}: \mu_{i}(R)-D=0\right\}=\left\{R \in R_{+}^{m}: R \geq \lambda^{i}, R_{j}=\lambda_{j i} \text { for some } j\right\} .
$$

Thus $Q_{i}$ is the boundary of the the cone, or orthant, $R \geq \lambda^{i}$.

Let us examine the conditions for there to exist certain steady states for (2.5). Of course, there is a trivial steady state with $N_{i}=0$ and $R_{i}=S_{i}$ for all $i$. There is a nontrivial steady state $\left(R^{*}, N^{*}\right)$ of $(2.5)$ with $N_{i}^{*}>0$ for $i \in I$ and $N_{i}^{*}=0$ for $i \neq I$ if and only if the polyhedral cone

$$
K_{I} \equiv\left\{R \in R_{+}^{m}: R=S-\sum_{i \in I} P_{i} c^{i}, P_{i}>0\right\}
$$

with vertex $S$ has a point $R^{*}$ in common with

$$
Q_{I} \equiv \cap_{i \in I} Q_{i} .
$$

For once $R^{*}$ is determined, then $N_{i}^{*}=P_{i}, i \in I$. We will make the generic assumption that the $\lambda$ are sufficiently different:

(H1) The entries in the each row of $\lambda$ are distinct.

This will ensure generic pair-wise intersections of $Q_{i}$ and $Q_{j}$ for $i \neq j$.

Let $M=\{1,2, \cdots, m\}$ and let $\# I$ denote the number of species in $I$. As $R \in Q_{I}$ implies $R \geq \lambda^{i}, i \in I$ and for each $i$ there exists $\sigma(i) \in M$, not necessarily unique, such that $R_{\sigma(i)}=\lambda_{\sigma(i) i}$, it follows that $\lambda_{\sigma(i) i}>\lambda_{\sigma(i) i^{\prime}}$ for each $i^{\prime} \in I, i^{\prime} \neq i$. In other words, each species $i \in I$ must be the inferior competitor among the species in $I$ for some resource $\sigma(i)$. Furthermore, the map $\sigma: I \rightarrow M$ must be injective since if $\sigma(i)=\sigma(p)$ for $i \neq p$, we have the contradiction:

$$
\lambda_{\sigma(i) i}>\lambda_{\sigma(i) p}=\lambda_{\sigma(p) p}>\lambda_{\sigma(p) i}=\lambda_{\sigma(i) i} .
$$

This reasoning leads to

$$
Q_{I}=\cup_{\sigma \in P_{I}}\left\{R \in R_{+}^{m}: R_{\sigma(i)}=\lambda_{\sigma(i) i}, i \in I, R_{j} \geq \max _{i \in I}\left\{\lambda_{j i}\right\}, j \notin \sigma(I)\right\}
$$

where $P_{I}$ is the possibly empty set of injective maps $\sigma: I \rightarrow M$ satisfying the property that for each $i \in I, \lambda_{\sigma(i) i}>\lambda_{\sigma(i) i^{\prime}}$ for each $i^{\prime} \in I, i^{\prime} \neq i$. In the case $P_{I}=\emptyset$ then $Q_{I}=\emptyset$. In particular, $Q_{I}=\emptyset$ if $\# I>m$ : there can be no steady state where the number of species present exceeds $m$, the number of resources. Also, $P_{I}=\emptyset$ if $\lambda^{i}>\lambda^{i^{\prime}}$ for some $i, i^{\prime} \in I$.

As $R^{*} \in K_{I}, R^{*}<S$ and as $R^{*} \in Q_{I}, R^{*} \geq \lambda^{i}, i \in I$. Hence a necessary condition for such a steady state to exists is that $S>\lambda^{i}$ for $i \in I$. 
If $Q_{I}$ is non-empty, then it is a union of portions of hyperplanes of dimension $m-\# I$. The cone $K_{I}$ has dimension equal to the dimension of $\operatorname{span}\left\{c^{i}: i \in I\right\}$. Hence we expect no steady state if $\operatorname{dim} \operatorname{span}\left\{c^{i}: i \in I\right\}<\# I$.

It seems unproductive to attempt a general result concerning the existence of steady states with arbitrary support $I$. Two special cases are easily dealt with, namely single-population steady states and positive steady states when the number of species equals the number of resources. We write $E_{i}$ for a single-population steady state for the $i$ th species where $I=\{i\}$ :

$$
E_{i}=\left(R^{i}, 0,0, \cdots, 0, N_{i}, 0, \cdots, 0\right)
$$

which exists provided $S>\lambda^{i}$. Here, the vector $R^{i}$ is determined simply as the point of intersection of the line through $S$ in the direction $c^{i}$ and the isocline $Q_{i}$ : $R^{i}=S-c^{i} N_{i} \in Q_{i}$. We will always assume that this line meets $Q_{i}$ generically, that is, at a point where, for some $j, R_{j}^{i}=\lambda_{j i}$ and $R_{k}^{i}>\lambda_{k i}, k \neq j$. In this case we say that species $i$ is limited by resource $j$. Hereafter, we assume that

(H2) $S>\lambda^{i}$ for each index $i$.

Otherwise the $i$ th species is eliminated due to lack of some resource.

Lemma 2.2 If $S_{j}<\lambda_{j i}$ for some $j$, then $\lim _{t \rightarrow \infty} N_{i}(t)=0$.

Proof Since $R_{j}^{\prime} \leq D\left(S_{j}-R_{j}\right)$ we see that for all large $t, R_{j}(t)<\lambda_{j i}$ and hence $\mu_{i}(R)-D<0$.

Below, we restrict attention to the special case $m=n$. There is a positive steady state if and only if (1) there is sufficient supply of each resource, (2) each species is the inferior competitor for exactly one resource, and (3) a positivity condition holds.

Theorem 2.3 Suppose $m=n$ in (2.5) and suppose that $C$ is non-singular. Then there exists at most one equilibrium $E^{*}=\left(R^{*}, N^{*}\right)$ where $N^{*}>0 . E^{*}$ exists if and only if

(a) $S>\lambda^{i}$ for $1 \leq i \leq n$. (A standing assumption)

(b) There exists a permutation $\sigma$ of $\{1,2, \cdots, n\}$ such that for each index $l$, $\lambda_{\sigma(l) l}>\lambda_{\sigma(l) k}$ for all $k \neq l$.

(c) The unique solution $N^{*}$ of $C N^{*}=S-R^{*}$, where $R^{*}$ is given below, satisfies $N^{*}>0$.

In fact, letting $\mu=\sigma^{-1}$ we have

$$
R^{*}=\left(\lambda_{1 \mu(1)}, \lambda_{2 \mu(2)}, \cdots, \lambda_{n \mu(n)}\right) \text { and } C N^{*}=S-R^{*}
$$

Species $\mu(j)$ is the inferior competitor on resource $j$. The proof is immediate from the considerations above.

Remark 2.4 If (b) holds we may renumber the resources such that $\sigma=$ identity; species $l$ is worst competitor for resource $l$. Of course, this rearranges the rows of $C$.

Remark 2.5 The existence of a positive $N^{*}$ satisfying $C N^{*}=S-R^{*}$ can be viewed geometrically. If $\Sigma_{n-1} \equiv\left\{x \in R_{+}^{n}: \sum x_{i}=1\right\}$ is the standard probability simplex, then $c^{i}$ belongs to the relative interior of $\Sigma_{n-1}, 1 \leq i \leq n$. A positive 
solution $N^{*}$ exists if and only if $S-R^{*} / 1_{m}^{T}\left(S-R^{*}\right)$ belongs to the relative interior of the convex hull of $\left\{c^{1}, \cdots, c^{n}\right\}$ on $\Sigma_{n-1}$.

Now we return to the reduced system (2.8). It has some very nice properties, chiefly, it is a competitive system on the cone $R_{+}^{n}$.

Lemma 2.6 The extended reduced system (2.8) on $R_{+}^{n}$ is a competitive system in the sense that its time-reversal satisfies the Kamke conditions and hence its forward flow (the backward flow of (2.8)) preserves the order relation $\leq$ on $R^{n}$. In fact, the time reversed flow is strongly order preserving in the interior of $R_{+}^{n}$ :

$$
N\left(t_{1}\right) \leq \tilde{N}\left(t_{1}\right), N\left(t_{1}\right) \neq \tilde{N}\left(t_{1}\right) \text { and } t_{0}<t_{1} \Longrightarrow N\left(t_{0}\right)<\tilde{N}\left(t_{0}\right)
$$

provided both solutions remain in the interior of $R_{+}^{n}$ on $\left[t_{0}, t_{1}\right]$. The interior of $R_{+}^{n}$ is an invariant set for (2.8). Furthermore, (2.8) is globally Lipschitz continuous $\left(C^{1}\right.$ if $\left.m=1\right)$ on $R_{+}^{n}$ and all its trajectories eventually enter and remain in $\Gamma$. (H2) implies that the origin is a repeller and each single-population steady state $E_{i}$ exists.

Proof We must show that the time-reversed system $N_{i}^{\prime}=-N_{i}\left(\mu_{i}(R)-D\right) \equiv$ $F_{i}(N), 1 \leq i \leq n$ where $R=S-C N$, satisfies the Kamke condition [33]: $N, \tilde{N} \in$ $R_{+}^{n}, N \leq \tilde{N}$ and $N_{i}=\tilde{N}_{i}$ for some $i$ implies that $F_{i}(N) \leq F_{i}(\tilde{N})$. As $R=S-C N \geq$ $\tilde{R}=S-C \tilde{N}$, we have $f_{j i}\left(R_{j}\right) \geq f_{j i}\left(\tilde{R}_{j}\right)$. We conclude that $\mu_{i}(R)-D \geq \mu_{i}(\tilde{R})-D$ and so $F_{i}(N) \leq F_{i}(\tilde{N})$. To prove strong monotonicity, we appeal to Theorem 4 of Walter where Lipschitzian systems are considered [36]. Accordingly, we must show that for every point $N^{0}>0$ and for each pair of nonempty disjoint index sets $\alpha, \beta$ with $\alpha \cup \beta=\{1,2, \cdots, n\}$ there are $j \in \alpha, k \in \beta$ and an open ball $B$ containing $N^{0}$ such that $F_{j}(N)$ is strictly increasing in $N_{k}$ for each $N \in B$. In fact, we claim that $F_{j}(N)$ is strictly increasing in $N_{k}, j \neq k$, for all $N>0$. Let $N>0$ and $N+t e_{k}$ where $t>0$ and $e_{k}$ is the standard basis vector having a one in the $k$ th component and all others zero. Then $\mu_{j}(S-C N)=f_{p j}\left(S_{p}-\sum_{l} c_{p l} N_{l}\right)$ for some $p$ and

$$
\begin{aligned}
\mu_{j}\left(S-C\left(N+t e_{k}\right)\right) & =\min _{r}\left\{f_{r j}\left(S_{r}-\sum_{l} c_{r l} N_{l}-t c_{r k}\right)\right\} \\
& \leq f_{p j}\left(S_{p}-\sum_{l} c_{p l} N_{l}-t c_{p k}\right) \\
& <f_{p j}\left(S_{p}-\sum_{l} c_{p l} N_{l}\right) \\
& =\mu_{j}(S-C N)
\end{aligned}
$$

where we have used the positivity of $c_{p k}$ and the strict monotonicity of $f_{p j}$ on all of $R$. Hence,

$$
F_{j}(N)=-N_{j}\left[\mu_{j}(S-C N)-D\right]<-N_{j}\left[\mu_{j}\left(S-C\left(N+t e_{k}\right)\right)-D\right]=F_{j}\left(N+t e_{k}\right) .
$$

since $N_{j}>0$. By Theorem 4 in [36], we have proved strong monotonicity. (H2) says $S>\lambda^{i}$ for all $i$ so $R=S-C N \approx S>\lambda^{i}$ for all $i$ when $N \approx 0$. Thus $\mu_{i}(R)-D>\epsilon>0$ for some small $\epsilon$ if $N$ is small.

Of course, competitive systems can have complicated $((n-1)$-dimensional) dynamics according to Smale [31]. Furthermore, (2.8) is not a $C^{1}$ vector field nor is $(2.5)$ unless $m=1$. The former fails to be $C^{1}$ on the union of finitely many 
$(n-1)$-dimensional surfaces. In the following we will want these bad surfaces to contain no steady states.

The point $\infty$ is a repeller, or source, for (2.8) in the sense that the "basin of repulsion" of $\infty$, the set $R(\infty)=\left\{N=N(0) \in R_{+}^{n}: N(t) \rightarrow \infty, t \rightarrow-\infty\right\}$, is non-empty and open relative to $R_{+}^{n}$. In fact, $R_{+}^{n} \backslash \Gamma \subset R(\infty)$ but as the origin is a repeller for $(2.8), R(\infty)$ does not intersect the basin of repulsion of the origin, itself a non-empty, relatively open neighborhood of the origin. Define

$$
\Sigma=\operatorname{clos}\left[\partial R(\infty) \cap \operatorname{Int}\left(R_{+}^{n}\right)\right]
$$

where the closure and interior are taken relative to $R^{n}$. A result of Hirsch [12] allows us to reduce the dimension of our problem still further.

Theorem 2.7 Assume that the right-hand side of (2.8) is $C^{1}$ in a neighborhood of each of its non-trivial steady states. Then $\Sigma$ is a closed $(n-1)$-cell, contained in $\Gamma$, which attracts all trajectories except the origin. $\Sigma$ is Lipschitz-homeomorphic to the standard $(n-1)$-probability simplex in $R_{+}^{n}$ under radial projection.

Proof This follows from the proof of Theorem 1.7 of Hirsch [12]. We have

$$
\frac{\partial\left(\mu_{i}-D\right)}{\partial N_{j}}(N *)=-f_{p i}^{\prime} c_{p j}<0
$$

for some index $p$ such that $\mu_{i}(R)=f_{p i}\left(R_{p}\right)$ at a steady state $N *$. A careful examination of the proof of the results in [12] reveals that the hypothesis that percapita growth rates are $C^{1}$ is used only for establishing (1) the strong monotonicity of the time reversed flow in the interior of $R_{+}^{n}$ and (2) that the origin is the only weak source other than the point at infinity. However, we have shown that (1) holds and (2) follows as in [12] by linearization at equilibria.

As a consequence of Theorem 2.7, we need only understand the dynamics of (2.8) on the "carrying simplex" $\Sigma$. Thus, we have obtained a remarkable reduction of dimension from the original system (2.5) of $n+m$ equations to dynamics on an $n-1$ dimensional attractor for (2.8). More precisely, we have the following result (recall we are assuming (H2) holds).

Theorem 2.8 Assume that the hypotheses of Theorem 2.7 hold. An omega limit set, $\Omega$, of a solution of (2.5) with $N(0) \neq 0$ can be described as follows

$$
\Omega=\{(R, N): R=S-C N, N \in A\}
$$

where $A \subset \Sigma$ is a compact, connected, invariant and chain recurrent set relative to (2.8).

Proof The only assertions that do not follow immediately from standard facts concerning omega limit sets are that $A \subset \Sigma, A$ is invariant and chain recurrent for (2.8). See $[6,30]$ for the definition of chain recurrence; we do not give it here since we use only one fact about such sets stated below. If $u \equiv S-R-C N$, then (2.5) becomes

$$
\begin{aligned}
N_{i}^{\prime} & =N_{i}\left[\mu_{i}(S-u(t)-C N)-D\right], 1 \leq i \leq n \\
u_{j}^{\prime} & =-D u_{j}, 1 \leq j \leq m
\end{aligned}
$$


where time $t$ is explicit in the first equation only since we will regard the above system as a non-autonomous one where $u(t)=u(0) e^{-D t}$. In fact, it is an asymptotically autonomous system, asymptotic to the autonomous "limiting" system

$$
\begin{aligned}
N_{i}^{\prime} & =N_{i}\left[\mu_{i}(S-C N)-D\right], 1 \leq i \leq n \\
u_{j}^{\prime} & =-D u_{j}, 1 \leq j \leq m .
\end{aligned}
$$

in the sense of [30]. Consequently, by Theorem 1.8 of [30], in the $(u, N)$ variables, $\Omega=\{0\} \times A$ is invariant and chain recurrent for the limiting system. Now a chain recurrent set $C$ cannot contain a proper subset which is a repeller for the dynamics restricted to $C$ [6]. Since $N(0) \neq 0, \Omega$ is not the singleton $\{(0,0)\}(\{(S, 0)\}$ in $(R, N)$ coordinates) by (H2), since then for large $t, N_{i}^{\prime} \approx N_{i}\left(\mu_{i}(S)-D\right)$ so $N_{i}(t)$ diverges. Therefore $\{(0,0)\}$ would be a proper subset of $\Omega$ if $(0,0)$ belonged to $\Omega$. But, in view of Lemma 2.6, $\{(0,0)\}$ is a repeller for the limiting system, restricted to $\Omega=\{0\} \times A$. We conclude that $0 \notin A$. Therefore, $A$ is invariant for (2.8) and does not contain the origin. By Theorem 2.7, $A \subset \Sigma$.

As an immediate consequence of Theorem 2.8, in case $n=1$ we have $(R(t), N(t)) \rightarrow$ $E_{1}$ as $t \rightarrow \infty$ if $N(0) \neq 0$.

A result of Hofbauer $[14,15]$ concerning the existence of saturated steady states will prove useful. In the context of (2.8), a steady state $N^{*}$ is said to be saturated if $\mu_{i}-D \leq 0$ if $N_{i}^{*}=0$, where, of course, $\mu_{i}$ is evaluated at $S-C N^{*}$. Observe that $N^{*}$ is trivially saturated if $N^{*}>0$; if $N_{i}^{*}=0$, then $\mu_{i}-D$ is an eigenvalue of the Jacobian of the vector field (should it exist) at $N^{*}$ and is non-positive if $N^{*}$ is saturated.

Theorem 2.9 If the hypotheses of Theorem 2.7 hold then there exists a saturated steady state of (2.8). If, in addition, every saturated steady state is nondegenerate, then the sum of their indices must be $(-1)^{n}$.

Proof Hofbauer proves this theorem under the smoothness hypotheses that the vector field is $C^{1}$. However, as he uses the Brouwer degree and the index theorem, he requires this only near each steady state.

Under the stronger conditions of Theorem 2.9, either there exists a positive steady state, or, there exists at least one boundary steady state which is stable (resistant) to invasion by any species not present.

In the special case that $m=n$, when the hypotheses (a)-(c) of Theorem 2.3 hold, we can linearize about the positive steady state of (2.8). Denote by $e_{i}$ the $i$ th standard basis vector with one in the $i$ th component.

Theorem 2.10 Let the hypotheses of Theorem 2.3 and conditions (a)-(c) hold. Then the linearization of (2.8) exists at the unique positive steady state $N^{*}$ and equals

$$
J\left(N^{*}\right)=-\operatorname{diag}\left[N_{1}^{*} f_{\sigma(1) 1}^{\prime}\left(\lambda_{\sigma(1) 1}\right), \cdots, N_{n}^{*} f_{\sigma(n) n}^{\prime}\left(\lambda_{\sigma(n) n}\right)\right] P C
$$

where $P$ is the permutation matrix with $i$-th row equal $e_{\sigma(i)}$. Thus, its determinant has the same sign as

$$
(-1)^{n} \operatorname{sign}(\sigma) \operatorname{det}(C)
$$

and one of its eigenvalues is simple and negative and has greater modulus than all others. If all saturated steady states are non-degenerate and $\operatorname{sign}(\sigma) \operatorname{det}(C)<0$ then there must exist a saturated equilibrium on the boundary. 
Proof If $N \approx N^{*}$ then $R \approx R^{*}$ given in (2.9). Now, $f_{j i}\left(R_{j}\right) \approx f_{j i}\left(\lambda_{j \mu(j)}\right)>$ $f_{j i}\left(\lambda_{j i}\right)=D$ for $j \neq \sigma(i)$ while $f_{j i}\left(\lambda_{j \mu(j)}\right)=D$ for $j=\sigma(i)$ because species $\mu(j)$ is the worst competitor for resource $j$. Consequently, $\mu_{i}(R)=f_{\sigma(i) i}(R)$ for $R \approx R^{*}$. It follows that $J\left(N^{*}\right)$ exists. The penultimate assertion follows from Perron-Frobenius Theorem since $C>0$. The final assertion follows from Theorem 2.9.

We will usually renumber species so that $\sigma$ is the identity, in which case its sign is one.

Remark 2.11 Suppose, as in Remark 2.4, that species have been numbered so that species $i$ is the inferior competitor for resource $i$. Then

$$
J\left(N^{*}\right)=-Q C,
$$

where $Q \equiv \operatorname{diag}\left[N_{1}^{*} f_{11}^{\prime}\left(\lambda_{11}\right), \cdots, N_{n}^{*} f_{n n}^{\prime}\left(\lambda_{n n}\right)\right]$. Because the determinant is the product of the eigenvalues, one easily sees that if $\operatorname{det}(C)<0$, then $J\left(N^{*}\right)$ has a positive eigenvalue so $N^{*}$ is unstable. On the other hand, if nonsingular matrix $C$ is diagonally dominant $\left(c_{i i} \geq \sum_{j \neq i} c_{i j}\right.$ all $\left.i\right)$ then the Gerschgorin Circle Theorem (see e.g. [32]) and nonsingularity imply that all eigenvalues of $J\left(N^{*}\right)$ have negative real part so $N^{*}$ is asymptotically stable. Diagonal dominance means roughly that resource $i$ makes up a larger fraction of species $i$ than it does for all other species combined. Alternatively, if $C$ is symmetric and positive definite, then so is $Q^{1 / 2} C Q^{1 / 2}$, which is similar to $Q C$ and hence has the same eigenvalues. It then follows that all eigenvalues of $J\left(N^{*}\right)$ are negative and $N^{*}$ is asymptotically stable. Likewise, if nonsingular matrix $C$ is totally nonnegative (all minors of all sizes are nonnegative) then $N^{*}$ is asymptotically stable for then $-J\left(N^{*}\right)$, being the product of two totally nonnegative matrices, is totally nonnegative so all its eigenvalues are nonnegative real numbers [7].

\section{Two Species}

An immediate consequence of Theorem 2.8 for the case of two species is the following result.

Theorem 3.1 Assume the hypotheses of Theorem 2.7 hold and that $n=2$. Then every solution of (2.5) converges to a steady state.

Proof $\Sigma$ is a one-dimensional curve connecting $E_{1}$ to $E_{2}$. A compact, connected, invariant and chain recurrent subset of it can only be a steady state for a chain recurrent set can have no proper attractor (repeller).

The case $m=n=2$ was the first non-trivial one to be considered. The outcomes of two-species competition for two resources are the same as those for the classical Lotka-Volterra model of competition: competitive exclusion, coexistence, and bistability.

Theorem $3.2([17,23,5])$ Let $m=n=2$. If species one is the worst competitor for both resources, then $E_{2}$ attracts all solutions satisfying $N(0)>0$. If species two is the worst competitor for both resources, then $E_{1}$ attracts all solutions satisfying $N(0)>0$. If each species is the worst competitor for one of the resources, then we may suppose species $i$ is the worst competitor for resource $i, i=1,2$. In this case, (H2) implies that $S$ belongs to the open quadrant $\left(\lambda_{11}, \lambda_{22}\right)+\operatorname{Int} R_{+}^{2}$ and we have the following outcomes: 
(a) If $S$ lies below the closed cone $\left(\lambda_{11}, \lambda_{22}\right)+C R_{+}^{2}$, then $E_{1}$ attracts all solutions with $N(0)>0$.

(b) If $S$ lies above the closed cone $\left(\lambda_{11}, \lambda_{22}\right)+C R_{+}^{2}$, then $E_{2}$ attracts all solutions with $N(0)>0$.

(c) If $S \in\left(\lambda_{11}, \lambda_{22}\right)+C$ Int $R_{+}^{2}$ and $\operatorname{det} C>0$, then the coexistence steady state attracts all solutions with $N(0)>0$.

(d) If $S \in\left(\lambda_{11}, \lambda_{22}\right)+C$ Int $R_{+}^{2}$ and $\operatorname{det} C<0$, then the coexistence steady state is a saddle point, $E_{1}$ and $E_{2}$ are local attractors with open basins of attraction whose union has full measure.

Proof This is only a sketch. If one species is the worst competitor on both resources then there can be no coexistence steady state by Theorem 2.3. Thus $\Sigma$ consists of $E_{1}, E_{2}$ and a single connecting trajectory. Now, supposing that species $i$ is worst for resource $i$ and that (a) or (b) hold, then there cannot be a coexistence steady state by Theorem 2.3 because (c) of that result fails. Again, $\Sigma$ consists of $E_{1}, E_{2}$ and a single connecting trajectory. That the local attractor is the global attractor for $N(0)>0$ in each case can be deduced by using the Butler-McGehee lemma (see e.g. [32]). In cases (c) and (d) a coexistence steady state exists by Theorem 2.3 and its stability, at least for (2.8), is determined by the trace and determinant of its Jacobian. As the trace is negative (see Theorem 2.10), it is an attractor in case (c) and a saddle in case (d). It follows that $\Sigma$ consists of $E_{1}, E_{2}$, the coexistence steady state, and two heteroclinic trajectories, converging on the coexistence steady state in (c) and diverging from it in case (d). Again, a ButlerMcGehee argument is required together with understanding the location of local stable manifolds.

\section{Three Species}

An immediate consequence of Theorem 2.8 for the case of three species is the following result.

Theorem 4.1 Assume the hypotheses of Theorem 2.7 hold and that $n=3$. Then an omega limit set of a solution of (2.5) which contains no steady state must contain a non-trivial periodic orbit. The existence of a non-trivial periodic orbit or a heteroclinic cycle of non-trivial equilibria implies the existence of a coexistence steady state and therefore, $m \geq 3$.

Proof $\Sigma$ is homeomorphic to the standard two-dimensional simplex with corners, the steady states $E_{1}, E_{2}$, and $E_{3}$. The remaining assertions follow from the Poincaré-Bendixson theory applied to the flow on $\Sigma$.

4.1 Two resources. For three species competing for only two resources there can be no coexistence steady states involving all species but there can be pairwise coexistence states $E_{i j}, i \neq j$ where only species $i$ and $j$ are present.

Theorem 4.2 ( $m=2[26])$ If $m=2$ and $n=3$, then every solution of (2.5) converges to one of the steady states $E_{i}$ or $E_{i j}$. In particular, for every initial data, at least one species is washed out; which species are washed out may be initial condition dependent.

Proof This, again is a sketch. As $m<n$ there can be no coexistence steady state. By Theorem 4.1, there can be no periodic orbits or heteroclinic cycles on $\Sigma$. 
Application of the Butler McGehee lemma implies that each omega limit set must be a single boundary steady state.

There are some 43 distinct phase portraits possible in this case, one of which is depicted in (figure 1) below. This phase portrait occurs for the following parameter restrictions:

$$
\begin{aligned}
& \lambda_{11}<\lambda_{12}<\lambda_{13}, \\
& \lambda_{23}<\lambda_{22}<\lambda_{21}, \\
& \frac{c_{21}}{c_{11}}<\frac{S_{2}-\lambda_{21}}{S_{1}-\lambda_{12}}<\frac{c_{22}}{c_{12}}, \\
& \frac{c_{21}}{c_{11}}<\frac{S_{2}-\lambda_{21}}{S_{1}-\lambda_{13}}<\frac{c_{23}}{c_{13}}, \\
& \frac{c_{22}}{c_{12}}<\frac{S_{2}-\lambda_{22}}{S_{1}-\lambda_{13}}<\frac{c_{23}}{c_{13}} .
\end{aligned}
$$

Only the phase portrait on $\Sigma$ is shown.

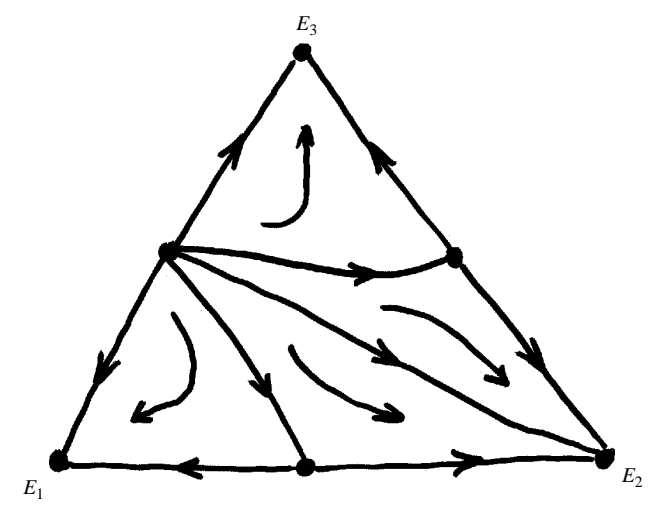

Figure 1 An example of tri-stability with attractors $E_{1}, E_{2}$, and $E_{3}$.

The authors failed to answer the question posed in the title of [26]: how many species can two resources support? Theorem 4.2 implies that two resources cannot support three species. We do not know if the same conclusion holds for four species but we did find a heteroclinic cycle among the four single-population steady states. This suggests the possibility of a bifurcation of a limit cycle from some heteroclinic cycle for four species on two resources.

4.2 Three resources. Several of the exciting conclusions of Huisman and Weissing $[19,20]$ stem from their numerical discovery of a limit cycle for the threeresource three-species model for certain parameters. The existence of this limit cycle was established in [27]. Here, we focus on this important special parameter region.

Let $e_{i j}$ denote the value of $\mu_{i}-D$ at $E_{j}$. For $i \neq j$, these are eigenvalues of the Jacobian of the vector field (2.8) at $E_{j}$. We make the following hypotheses for 
the case $m=n=3$.

$$
\begin{gathered}
S_{1}>\lambda_{11}>\lambda_{12}>\lambda_{13}, \\
S_{2}>\lambda_{22}>\lambda_{23}>\lambda_{21}, \\
S_{3}>\lambda_{33}>\lambda_{31}>\lambda_{32} . \\
\frac{S_{1}-\lambda_{11}}{S_{2}-\lambda_{23}}>\frac{c_{13}}{c_{23}}, \quad \frac{S_{1}-\lambda_{11}}{S_{3}-\lambda_{31}}>\frac{c_{11}}{c_{31}}, \\
\frac{S_{2}-\lambda_{22}}{S_{1}-\lambda_{12}}>\frac{c_{22}}{c_{12}}, \quad \frac{S_{2}-\lambda_{22}}{S_{3}-\lambda_{31}}>\frac{c_{21}}{c_{31}}, \\
\frac{S_{3}-\lambda_{33}}{S_{1}-\lambda_{12}}>\frac{c_{32}}{c_{12}}, \quad \frac{S_{3}-\lambda_{33}}{S_{2}-\lambda_{23}}>\frac{c_{33}}{c_{23}} . \\
e_{12} e_{23} e_{31}+e_{13} e_{21} e_{32}>0 . \\
c_{11} c_{22}<c_{12} c_{21}, \\
c_{11} c_{33}<c_{13} c_{31}, \\
c_{22} c_{33}<c_{23} c_{32} .
\end{gathered}
$$

Conditions (4.1) and (4.2) reflect the following observation made by Huisman and Weissing [19]: there is a cyclic replacement of species if each species is an intermediate competitor for the nutrients that most limits its growth rate. If (4.1) and (4.2) hold, then $e_{21}, e_{32}, e_{13}>0$ and $e_{12}, e_{23}, e_{31}<0$ so there exists a heteroclinic cycle for $(2.8)$

$$
E_{1} \rightarrow E_{2} \rightarrow E_{3} \rightarrow E_{1}
$$

on the boundary of $R_{+}^{3}$ bounding $\Sigma$. Consequently, $E^{*}$ exists by Theorem 4.1 or by Theorem 2.9. (4.3) ensures that the heteroclinic cycle is a repeller so the reduced system (2.8) is uniformly persistent. See Hofbauer and Sigmund [15] for detailed discussions on (4.3). (4.4) is the condition for $E^{*}$ to be unstable (a saddle with one dimensional stable manifold).

Theorem 4.3 ([27]) Suppose that $m=n=3$ and the hypotheses (4.1)-(4.4) are satisfied. Then the heteroclinic cycle (4.5) exists and is repelling on $\Sigma$. $E^{*}$ exists and its stable manifold is a curve, totally ordered by $<$, connecting the origin and point at infinity to $E^{*}$. Moreover every solution of (2.8) initiating at an interior point of $R_{+}^{3}$ not on the stable manifold of $E^{*}$ approaches a nontrivial positive periodic orbit.

Proof See [27] for the local stability calculation for $E^{*}$ and that $e_{21}, e_{32}, e_{13}>0$ and $e_{12}, e_{23}, e_{31}<0$. As noted above, (4.3) and a result of Hofbauer [15] imply that the heteroclinic cycle is a repeller so (2.8) is (uniformly) persistent. Let $N(t)$ be a solution of (2.8) such that $N(0)$ lies in the interior of $R_{+}^{3}$ but not on the stable manifold of $E^{*}$. Since the system is persistent, its limit set lies in the interior of $R_{+}^{3}$. By Theorem 3.4.2 in Smith [33], the limit set of $N(t)$ is a nontrivial periodic orbit. The assertions concerning the stable manifold of $E^{*}$ follow from applying Theorem 4.3.4 of [33] to the time reversed (2.8).

Of course, $E^{*}$, its two-dimensional unstable manifold, at least one periodic orbit and the heteroclinic cycle (4.5) belong to the carrying simplex $\Sigma$. $E^{*}$ is the only point of intersection of the stable manifold of $E^{*}$ with $\Sigma$ as the latter is unordered [12]. Numerical evidence suggests the uniqueness of the limit cycle. In this case, the phase portrait on $\Sigma$ is as depicted in figure 2 . 


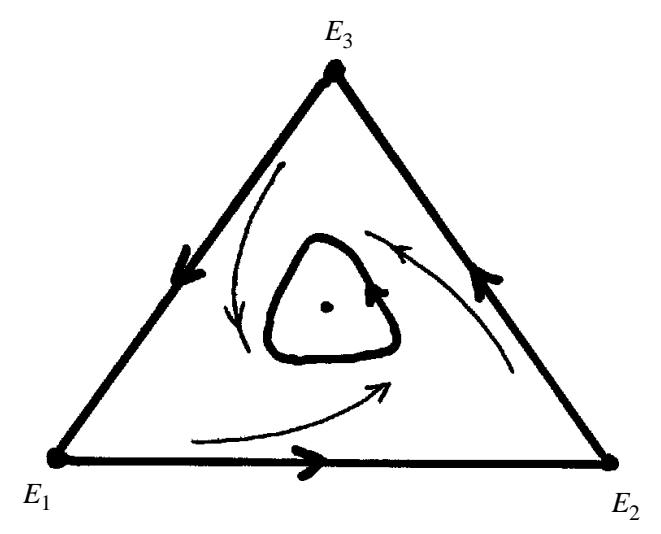

Figure 2 phase portrait on $\Sigma$.

Theorem 4.3 describes the dynamics of the reduced system (2.8) while the following focuses on the original system.

Theorem 4.4 Assume the hypotheses of Theorem 4.3 hold. Then there exists $\epsilon>0$ such that

$$
\liminf _{t \rightarrow \infty} N_{i}(t)>\epsilon, 1 \leq i \leq 3
$$

for every solution $(R(t), N(t))$ of (2.5) satisfying $N(0)>0$. In fact, except for those initial data belonging to the 4-dimensional stable manifold of $E^{*}$ (a set of measure zero in $\left.R^{6}\right)$, its omega limit set contains a non-trivial periodic orbit.

Proof The omega limit set $\Omega$ is described in Theorem 2.8 where $A \subset \Sigma$. But the heteroclinic cycle is a repeller for both (2.5) and for (2.8) by Corollary 2 of [13] (see also Theorem 12.2.1 of [15]) and therefore, (4.6) holds (for both systems). To see this, we employ the average Lyapunov function $P=\prod_{i=1}^{3} N_{i}^{p_{i}}$ where the $p_{i}>0$ are chosen so that $\dot{P} / P=\sum_{i} p_{i}\left(\mu_{i}-D\right)>0$ at the trivial steady state and each $E_{i}$. As is shown in [15], condition (4.3) is precisely the condition that this holds at the $E_{i}$ and since $\mu_{i}-D>0$ at the trivial steady state by (H2), the condition at this point is obvious. Note that dissipativity of (2.5) and of (2.8) is required for the applicability of Corollary 2 of [13].

By virtue of (4.6), $A$ is bounded away from the boundary of $\Sigma$ by a positive constant independent of positive initial data. As $A$ is chain recurrent, it cannot contain a proper subset which is a repeller for the dynamics restricted to itself. Recall that a neighborhood of $E^{*}$ in $\Sigma$ consists of its local unstable manifold relative to (2.8). Thus, either $A=\left\{E^{*}\right\}$ or $E^{*} \notin A$. The former case implies that the initial data belong to the stable manifold of $E^{*}$. Finally, as noted in Theorem 4.1, if $E^{*} \notin A$ then $A$ contains a periodic orbit.

The parameter values used in [20] satisfy (4.1)-(4.4). It is interesting to note that in this case species are cooperative to survive. As a matter of fact, if one of three competitors is absent, the existence of heteroclinic cycle indicates that one of the other two rivals can not survive. 
If the hypothesis (4.4) is replaced by

$$
\begin{aligned}
& c_{11} c_{22}>c_{12} c_{21}, \\
& c_{11} c_{33}>c_{13} c_{31}, \\
& c_{22} c_{33}>c_{23} c_{32}, \\
& 2 c_{11} c_{22} c_{33}>c_{13} c_{21} c_{32}+c_{12} c_{23} c_{31},
\end{aligned}
$$

then $E^{*}$ is asymptotically stable (see [27]). In this case, it is possible that $E^{*}$ is a global attractor when (4.3) holds.

\section{References}

[1] R. A. Armstrong and R. McGehee, Competitive exclusion, Amer. Natur., 115 (1980), pp. 151-170.

[2] M. M. Ballyk and G. S. K. Wolkowicz, An examination of the thresholds of enrichment: a resouce-based growth model, J. Math. Biol., 33 (1995), pp. 435-457.

[3] O. Baker, Who will win, Scientific American, May 2001.

[4] G. J. Butler and G. S. K. Wolkowicz, A mathematical model of the chemostat with a general class of functions describing nutrient uptake, SIAM J. Appl. Math., 45 (1985), pp. 138-151.

[5] G. J. Butler and G. S. K. Wolkowicz, Exploitative competition in a chemostat for two complementary, and possible inhibitory, resources, Math. Biosci., 83 (1987), pp. 1-48.

[6] C. Conley, Isolated invariant sets and the Morse index, CBMS Regional Conf. Ser. in Math. 38, Amer. math. Soc., Providence R.I., 1978.

[7] S. Fallet, Bidiagonal factorizations of totally nonnegative matrices, Amer. Math. Monthly 108 (2001), pp. 697-712.

[8] W.T. De Groot, Modelling the multiple nutrient limitation of algal growth, Ecological Modelling 18 (1983), 99-119.

[9] J.P. Grover, Resource Competition, Population and Community Biology Series, 19, Chapman\&Hall, New York, 1997.

[10] J. K. Hale, "Ordinary Differential Equations", Krieger, Malabar, FL, 1980.

[11] M.W.Hirsch, Systems of differential equations which are competitive or cooperative. I: limit sets, SIAM J.Appl. Math. 13 (1982), pp. 167-179.

[12] M.W.Hirsch, Systems of differential equations which are competitive or cooperative. III: Competing species, Nonlinearity 1 (1988), pp. 51-71.

[13] J. Hofbauer, A unified approach to persistence, Acta Appl. Math. (1989) 14, 11-22.

[14] J. Hofbauer, An index theory for dissipative semiflows, Rocky Mountain J. Math. 20 (1990), pp. 1017-1031.

[15] J. Hofbauer and K. Sigmund, The Theory of Evolution and Dynamical Systems, London Math. Soc. Student Texts 7, Cambridge University Press, Cambridge, 1988.

[16] S. B. Hsu, Limiting behavior for competing species, SIAM J. Appl. Math., 34 (1978), pp. 760-763.

[17] S. B. Hsu, K. S. Cheng, and S. P. Hubbell, Exploitative competition of microorganism for two complementary nutrients in continuous culture, SIAM J. Appl. Math., 41 (1981), pp. 422-444.

[18] S. B. Hsu, S. Hubbell and P. Waltman, A mathematical theory of single-nutrient competition in continuous cultures of micro-organisms, SIAM J. Appl. Math., 32 (1977), pp. 366-383.

[19] J. Huisman and F.J. Weissing, Biodiversity of plankton by species oscillations and chaos, Nature ,vol 402, 25 Nov. 1999, pp.407-410.

[20] J. Huisman and F.J. Weissing, Fundamental unpredictability in multispecies competition, Amer. Naturalist 157 (2001), 488-494.

[21] J. Huisman and F.J. Weissing, Coexistence and resource competition, Nature 407, 694, 2000.

[22] J. Huisman and F.J. Weissing, Biological conditions for oscillations and chaos generated by multispecies competition, Ecology 82(10), (2001) p.2682-2695.

[23] J. A. Leon and D. B. Tumpson, Competition between two species for two complementary or substitutable resources, J. Theor. Biol., 50 (1975), pp. 185-201.

[24] B. Li, Global asymptotic behaviour of the chemostat; general response functions and different removal rates, SIAM J. Appl. Math., (1999), pp. 411-422.

[25] B. Li, Analysis of Chemostat-Related Models With Distinct Removal Rates, Ph.D thesis, Arizona State University, 1998. 
[26] B. Li and H.L. Smith, How many species can two essential resources support?, SIAM J. Appl. Math.62, 2001, 336-66.

[27] B. Li, Periodic coexistence in the chemostat with three species competing for three essential resources, Math. Biosciences 174, 2001,p. 27-40.

[28] P. Lundberg, E. Ranta, V. Kaitala, N. Jonzen, Coexistence and resource competition, Nature 407, 694, 2000.

[29] R.M. May and W.J.Leonard, Nonlinear aspects of competition between three species, SIAM J. Appl. Math. 29, 1975, 243-253.

[30] K.Mischiakow, H.L. Smith, H.Thieme, Asymptotically Autonomous semiflows: chain recurrence and Lyapunov functions, Trans. A.M.S. 347, 1995, 1669-1685.

[31] S. Smale, On the differential equations of species in competition, J. Math. Biol. 3 (1976), 5-7.

[32] H.L. Smith and P. Waltman, "The Theory of the Chemostat," Cambridge University Press, 1995.

[33] H. L. Smith, "Monotone Dynamical Systems, An Introduction to the Theory of Competitive and Cooperative Systems", Mathematical Surveys and Monographs 41, Amer. Math. Soc., Providence, RI, 1995.

[34] H.L.Smith, Periodic orbits of competitive and cooperative systems, J. Diff. Eqns. 65 (1986),pp. 361-373.

[35] D. Tilman, Resource competition and Community Structure, Princeton U. P., Princeton, N.J., 1982.

[36] W.Walter, On strongly monotone flows, Annales Polonici Mathematici, LXVI (1997), 269274.

[37] G. S. K. Wolkowicz and Z. Lu, Global dynamics of a mathematical model of competition in the chemostat: general response function and differential death rates, SIAM J. Appl. Math., 52 (1992), pp. 222-233. 